NOTA

\title{
OCORRÊNCIA DE Hyblaea puera (CRAMER, 1777) (LEPIDOPTERA: HYBLAEIDAE) EM TECA NO BRASIL ${ }^{(1)}$
}

\author{
OTÁVIO PERES-FILHO(2); ALBERTO DORVAL ${ }^{(2)}$; EVÔNEO BERTI-FILHO ${ }^{(3)}$
}

\begin{abstract}
RESUMO
Relata-se a ocorrência de Hyblaea puera (Cramer, 1777) em povoamentos homogêneos de teca, Tectona grandis L.f., no Brasil. Infestações dessa praga foram observadas em reflorestamentos localizados nos municípios de Cáceres e Rosário Oeste, Estado de Mato Grosso. Cita-se, também, a ocorrência deste lepidóptero em outras localidades brasileiras: Chapada dos Guimarães (MT), Nobres (MT), Jataí (GO), Dourados (MS) e Rio de Janeiro (RJ).

Palavras-chave: Insecta, desfolhador da teca, lagarta desfolhadora.
\end{abstract}

\author{
ABSTRACT \\ OCCURRENCE OF THE TEAK DEFOLIATOR Hyblaea puera (CRAMER, 1777) \\ (LEPIDOPTERA: HYBLAEIDAE) IN TEAK (Tectona grandis L.f.) IN BRAZIL
}

The occurrence of the Hyblaea puera (Cramer, 1777) in homogeneous plantations of teak, Tectona grandis L.f., in Brazil, is reported here. Infestations of this pest were observed in the districts of Cáceres and Rosário Oeste, State of Mato Grosso, Brazil. The occurrence of this moth is also registered in other Brazilian areas such as: Chapada dos Guimarães and Nobres (State of Mato Grosso), Jataí (State of Goiás), Dourados (State of Mato Grosso do Sul) and Rio de Janeiro (State of Rio de Janeiro).

Key words: Insecta, teak defoliator, defoliator caterpillar.

\section{INTRODUÇÃO}

A teca, Tectona grandis L.f., é uma espécie florestal originária da Índia, Tailândia, Burma, Camboja e Indonésia, cujas áreas de cultivo vêm se expandindo no Estado de Mato Grosso, em vista do alto valor comercial de sua madeira e por apresentar boa adaptabilidade em diferentes regiōes do Estado.

$\mathrm{Na}$ Índia, uma das principais pragas da teca é a lagarta-desfolhadora Hyblaea puera (Cramer). Essa praga encontra-se, principalmente, associada à teca, mas pode ocorrer em outras plantas como Vitex, Clerodendron,
Spathodea, Vitex altissima, Avicennia officinalis, Callicarpa arborea, V. peduncularis, V. pubescens, V. negundo e Oroxylum indicum (COMMONWEALTH InstituTe of Entomology, 1981; Mohanadas, 1986; BaKsha e Crawley, 1995).

No Estado de Mato Grosso, constatou-se a ocorrência de Hyblaea puera, pela primeira vez, em plantios homogêneos de teca nos municípios de Cáceres e Rosário Oeste, em 1997 e 1998, respectivamente, no início da estação chuvosa (outubro). Segundo BECKER ${ }^{(4)}$, em 1998, foram identificados exemplares de $H$. puera provenientes da Chapada dos Guimarães (MT), Nobres

$\left.{ }^{1}\right)$ Recebido para publicação em 18 de julho de 2001 e aceito em 28 de fevereiro de 2002.

$\left({ }^{2}\right)$ Faculdade de Engenharia Florestal, Universidade Federal de Mato Grosso, Av. Fernando Corrêa, s/n, 78060-900 Cuiabá (MT). E-mail: o.peres@zaz.com.br e adorval@zaz.com.br

$\left({ }^{3}\right)$ Departamento de Entomologia, Fitopatologia e Zoologia Agrícola, ESALQ/USP, Caixa Postal 9, 13418-900 Piracicaba (SP). E-mail: e.berti@carpa.ciagri.usp.br .

( $\left.{ }^{4}\right)$ BECKER, V. Universidade Nacional de Brasília. Comunicação pessoal, 1998. 


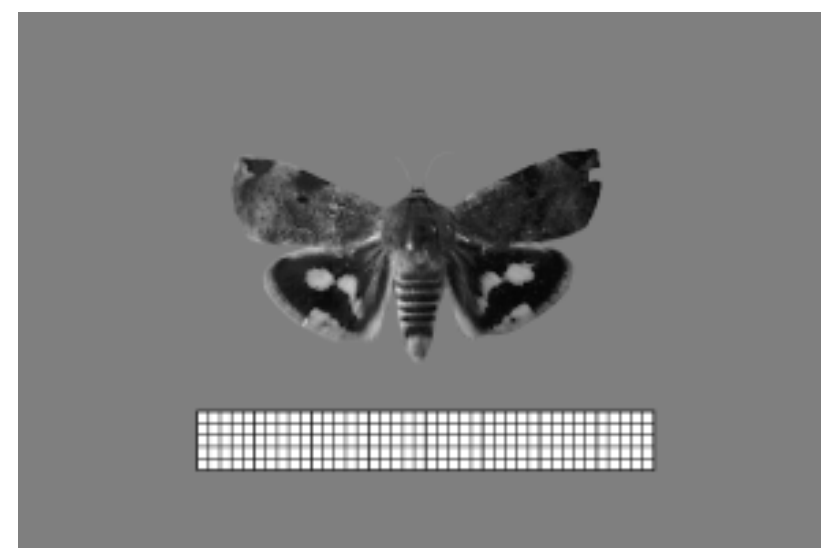

(MT), Jataí (GO), Dourados (MS) e Rio de Janeiro (RJ), evidenciando a grande disseminação dessa espécie pelo território brasileiro. Essa expansão apóia-se na hipótese de ocorrerem diversas espécies, arbóreas ou não, pertencentes à família Verbenaceae e também pelo hábito migratório das mariposas. BHOWMICK e VAISHAMPAYAN (1986) e Vaishampayan et al. (1987) observaram que, na Índia, os hábitos migratórios de $H$. puera estão associados às monções de sudoeste.

$\mathrm{O}$ fato de $H$. puera iniciar seus ataques no início do período chuvoso pode estar, provavelmente, correlacionado com a época de maior disponibilidade de folhas jovens. Segundo Murugan e Kumar (1996), $H$. puera apresentou maior quantidade de ovos e maior longevidade quando as lagartas foram alimentadas com folhas jovens, e uma redução nas eficiências de ingestão e digestão, quando alimentadas com folhas senescentes. NAIr et al. (1985), em experimento desenvolvido durante quatro anos em Kerala (Índia), observaram que a ocorrência de $H$. puera foi maior nos meses chuvosos.

Os exemplares de $H$. puera, coletados em Cáceres e Rosário Oeste, encontram-se depositados na coleção de referência do Laboratório de Proteção Florestal, da Faculdade de Engenharia Florestal, da Universidade Federal de Mato Grosso, em Cuiabá (MT).

\section{REFERÊNCIAS BIBLIOGRÁFICAS}

BAKSHA, M.W.; CRAWLEY, M.J. Relative preference of different host plants to teak defoliator, Hyblaea puera Cram. (Hyblaeidae: Lepidoptera) in Bangladesh. Bangladesh Journal of Forest Science, Bengladesh, v.24, n.1, p.21-25, 1995.

BHOWMICK, A.K.; VAISHAMPAYAN, S.M. Observation on the activity of teak defoliator Hyblaea puera Cramer on teak (Tectona grandis) influenced by the movement of monsoon. Journal of Tropical Forest Science, Kuala Lumpur, v.2, n.1, p.27-35, 1986.

COMMONWEALTH INSTITUTE OF ENTOMOLOGY. Lymantria dispar (L.), Hyblaea puera (Cram.): Distribution Maps of Pests. London, 1981. v.26, 2p,

MOHANADAS, K. A new host record for the teak defoliator, Hyblaea puera (Lepidoptera, Hyblaeidae). Current Science, Bangalore, v.55, n.23, p.1207-1208, 1986.

MURUGAN, K.; KUMAR, N.S. Host plant biochemical diversity, feeding, growth and reproduction of teak defoliator Hyblaea puera (Cramer) (Lepidoptera: Hyblaeidae). Indian Journal of Forestry, Dehra Dun, v.19, n.3, p.253-257, 1996.

NAIR, K.S.S.; SUDHEENDRAKUMAR, V.V.; VARMA, R.V.; CHACKO, K.C. Studies on the seasonal incidence of defoliators and the effect of defoliation on volume increment of teak. Kerala: Forest Research Institute, 1985. 78p. (Research Report, n. 30).

VAISHAMPAYAN, S.M.; VERMA, R.; BHOWMIK, A.K. Possible migration of teak-efoliator, Hyblaea puera Cramer (Lepidoptera: Hyblaeidae) in relation to the movement of the south-wet monsoon as indicate by light trap catches. Indian Journal of Agricultural Sciences, New Delhi, v.57, n.1, p.41-46, 1987. 